\title{
Tratamento cirúrgico da fratura multifragmentada da diáfise da clavícula pela técnica minimamente invasiva: Descrição e resultados preliminares*
}

\section{Surgical Treatment of Comminuted Midshaft Clavicle Fracture by Minimally Invasive Technique: Description and Preliminary Results}

\author{
Adriano Fernando Mendes Junior ${ }^{1(0)}$ Jose da Mota Neto ${ }^{1(0)}$ Igor Gerdi Oppe ${ }^{1(1)}$ \\ Leandro Furtado de Simoni ${ }^{2(1)}$ Vincenzo Giordano ${ }^{3(0)}$ Pedro José Labronici ${ }^{4}{ }^{(0)}$
}

${ }^{1}$ Hospital Universitário, Universidade Federal de Juiz de Fora, Juiz de Fora, MG, Brasil

Endereço para correspondência Adriano Fernando Mendes Junior,

2 Rua Sampaio, 468, apartamento 1402, Juiz de Fora, Minas Gerais, 36010-360, Brasil (e-mail: adrianofmjr@yahoo.com.br).

${ }^{3}$ Hospital Municipal Miguel Couto, Rio de Janeiro, RJ, Brasi

${ }^{4}$ Universidade Federal Fluminense, Niterói, RJ, Brasil

Rev Bras Ortop 2021;56(4):490-496.

\begin{abstract}
Resumo
Palavras-chave

- clavícula

- fraturas ósseas

- resultado do tratamento

- procedimentos cirúrgicos minimamente invasivos

Objetivo Avaliar os resultados funcionais e radiográficos do tratamento cirúrgico realizado em um grupo de pacientes com fratura multifragmentada da diáfise de clavícula, pela técnica minimamente invasiva, em seguimento mínimo de 12 meses. Métodos Estudo observacional longitudinal de 32 pacientes consecutivos (31 do sexo masculino, idade média 41 anos) com fratura multifragmentada da diáfise da clavícula tratados cirurgicamente pela técnica minimamente invasiva de osteossíntese com placa de reconstrução de $3,5 \mathrm{~mm}$ na posição superior, avaliados clínica e radiologicamente, com seguimento mínimo de 1 ano

Resultados Trinta pacientes (93,72\%) evoluíram com consolidação da fratura em tempo médio de 17 semanas (entre 12 e 24 semanas). 0 tempo de seguimento médio foi de 21 meses (variando de 12 a 45 meses). Não houve quebra de implantes ou pseudoartroses. Não houve queixa de parestesia na região das incisões cirúrgicas. $\mathrm{O}$ ombro tratado cirurgicamente apresentou menor elevação passiva e maior comprimento da clavícula $(p<0,05)$ em relação ao contralateral. Na avaliação funcional, encontramos um valor médio de Disfunções do Braço, Ombro e Mão (DASH, na sigla em inglês) $=1,75$, sendo o mesmo considerado satisfatório. Idade $>60$ anos apresentou correlação negativa com escore DASH $(p<0,05)$.
\end{abstract}

\footnotetext{
Trabalho desenvolvido no Hospital Universitário da Universidade Federal de Juiz de Fora, Juiz de Fora, MG, Brasil.
}

recebido

01 de Novembro de 2019

aceito

05 de Maio de 2020

Publicado on-line

Setembro 24, 2020
DOI https://doi.org/ $10.1055 / \mathrm{s}-0040-1714226$ ISSN 0102-3616. (c) 2020. Sociedade Brasileira de Ortopedia e Traumatologia. All rights reserved.

This is an open access article published by Thieme under the terms of the Creative Commons Attribution-NonDerivative-NonCommercial-License, permitting copying and reproduction so long as the original work is given appropriate credit. Contents may not be used for commercial purposes, or adapted, remixed, transformed or built upon. (https://creativecommons.org/ licenses/by-nc-nd/4.0/)

Thieme Revinter Publicações Ltda., Rua do Matoso 170, Rio de Janeiro, RJ, CEP 20270-135, Brazil 


\begin{abstract}
Keywords

- clavicle

- fractures, bone

- treatment outcome

- minimally invasive surgical procedures

Objective The present paper aimed to evaluate functional and radiographic outcomes from a group of patients with comminuted midshaft clavicle fracture who were surgically treated using a minimally invasive technique and followed-up for a minimum period of 12 months.

Methods Longitudinal, observational study with 32 consecutive patients (31 males; mean age, 41 years old) with comminuted midshaft clavicle fracture who were surgically treated using the minimally invasive osteosynthesis technique with a 3.5$\mathrm{mm}$ reconstruction plate in the upper position. Patients were clinically and radiologically evaluated for a minimum follow-up period of 12 months.

Results In 30 patients (93.72\%), fracture consolidation occurred in an average time of 17 weeks (range, 12 to 24 weeks). The mean follow-up time was 21 months (range, 12 to 45 months). No implant break or pseudoarthrosis were recorded. There was no complaint of paresthesia around the surgical incisions. The surgically-treated shoulder presented lower passive elevation and longer clavicle length $(p<0.05)$ compared with the contralateral shoulder. Functional evaluation revealed an average Disability of Arm, Shoulder and Hand (DASH) score of 1.75, which is considered satisfactory. Age $>60$ years old had a negative correlation with DASH score $(p<0.05)$.

Conclusion The minimally invasive osteosynthesis technique was satisfactory for the treatment of comminuted midshaft clavicle fracture, with a high consolidation rate and a low complication rate.
\end{abstract}

Conclusão A técnica minimamente invasiva de osteossíntese mostrou-se satisfatória para o tratamento da fratura multifragmentada da diáfise da clavícula, com elevada taxa de consolidação e baixo índice de complicações.

\section{Introdução}

As fraturas da clavícula correspondem a $2,6 \%$ a $5 \%$ de todas as fraturas nos adultos, ${ }^{1-3}$ e $\sim 80$ a $85 \%$, ocorrem no terço médio. ${ }^{1,2}$ A fratura complexa ou multifragmentada da diáfise da clavícula é comumente originada de acidentes de alta energia, trauma direto ou compressão axial. ${ }^{3,4} \mathrm{~A}$ clavícula apresenta biomecânica diferente de ossos longos, e o comportamento de uma fratura multifragmentada desse osso é pouco estudado. ${ }^{5}$ A literatura descreve que o tratamento conservador dessas fraturas associa-se a maiores índices de pseudoartrose. $^{2,6,7}$ Outros estudos argumentam que o tratamento cirúrgico melhora o desfecho funcional, quando comparado ao tratamento não cirúrgico. ${ }^{6-9}$ Em nosso meio, a maioria dos ortopedistas indica osteossíntese para fraturas diafisárias desviadas e/ou multifragmentadas. ${ }^{10}$

O método mais utilizado de osteossíntese das fraturas diafisárias desviadas da clavícula é a redução aberta e fixação interna com placa e parafusos. ${ }^{5,9}$ Porém, nas fraturas multifragmentadas, esta opção, por necessitar de extenso acesso ao foco de fratura, pode associar-se a altos índices de complicações como cicatrizes hipertróficas e dolorosas, ${ }^{4}$ infecção, ${ }^{11}$ pseudoartrose, ${ }^{12}$ falha do implante e refratura após a retirada do implante. ${ }^{13}$ Como vantagens, a técnica minimamente invasiva de osteossíntese (MIO) com placa preserva o suprimento sanguíneo no local de fratura ${ }^{14} \mathrm{e}$ pode diminuir estas complicações. A MIO é comumente usada nas fraturas complexas de ossos longos dos membros inferiores, ${ }^{15}$ além de possuir aplicabilidade comprovada em fraturas diafisárias dos membros superiores. ${ }^{16}$

Alguns autores descreveram suas técnicas e resultados de MIO em fraturas multifragmentadas da clavícula. ${ }^{17,18}$ Os estudos publicados utilizam implantes pouco acessíveis a população de nosso país que são usuários do Sistema Único de Saúde (SUS). Na literatura brasileira, não há estudos sobre a técnica MIO com placa para tratamento desse tipo de fratura. O objetivo do presente estudo é avaliar clínica e radiograficamente um grupo de pacientes com fratura multifragmentada da diáfise da clavícula tratados cirurgicamente através da técnica MIO com placa de reconstrução de $3.5 \mathrm{~mm}$ na posição superior.

\section{Metodologia}

Estudo observacional longitudinal, com levantamento inicial retrospectivo de pacientes com fratura multifragmentada da diáfise da clavícula tratados cirurgicamente pela técnica MIO com placa superior por um dos autores pesquisadores no período de janeiro de 2014 a maio de 2017, no hospital universitário da nossa instituição. 0 tamanho amostral de indivíduos corresponde ao número de pacientes que foram tratados cirurgicamente no período citado e que compareceram à avaliação. Foram incluídos no estudo pacientes com idade $>16$ anos, com fratura multifragmentada da diáfise da clavícula, tipo 2 B2 da classificação de Robinson, ${ }^{9}$ tratados cirurgicamente em até 21 dias após o trauma, que apresentavam seguimento mínimo de 1 ano. Foram excluídos 
pacientes com fraturas expostas ou com lesões vasculares e nervosas associadas, fraturas com extensão articular, fraturas e/ou luxações concomitantes com traumas da cintura escapular, associação com fraturas de outros segmentos do membro superior (braço, antebraço, punho e mão), história de fraturas prévias na clavícula ou traumatismos prévios na cintura escapular, fraturas patológicas, pacientes com doenças metabólicas e/ou congênitas. A amostra final foi composta por 32 pacientes. Os pacientes elegíveis ao estudo, após análise dos critérios de inclusão e exclusão, foram contatados para entrevista, explicação do estudo e apresentação do termo de consentimento livre e esclarecido (TCLE) da pesquisa, e aqueles que concordaram foram submetidos prospectivamente à avaliação clínica, ao questionário Disfunções do Braço, Ombro e Mão (DASH, na sigla em inglês) ${ }^{19}$ e a exames radiográficos. Avaliação funcional com aplicação do questionário DASH e exame físico (amplitude de movimento passiva do ombro, testes de impacto subacromial, manguito rotador, acromioclavicular, discinesia escápulo torácica, e força, aferida no movimento para elevação ativa, com auxílio de dinamômetro manual Science SuplySolutions\#U40812 [Science Supply Solutions, LLC, Bensenville, Illinois, United States of America], (graduação $1 \mathrm{~kg} / 10 \mathrm{~N}$ ) foi realizada por um examinador não envolvido nos procedimentos cirúrgicos como cirurgião principal. A avaliação radiográfica digital das clavículas em anteroposterior (AP) e craniocaudal modificada, para verificação do pós-operatório, e tórax pósteroanterior (PA), foi realizada para medida do comprimento final da clavícula, conforme critérios de Smekal et al. ${ }^{20}$ Por fim, foi feita a análise dos prontuários sobre desfechos ao ato cirúrgico, como tempo de consolidação, retardo de consolidação, pseudoartrose, infecção, soltura do implante, falha de material de síntese, dor residual e arco de movimento.

Técnica cirúrgica (adaptada de Jung et al. $^{18}$ ): paciente em posição cadeira de praia, com auxílio da radioscopia nas incidências frontal da clavícula e crânio caudal modificada com inclinação aproximada de $70^{\circ}$ (- Figura 1). Foi realizada manobra de redução da fratura ("Manobra de Kibler") com o cirurgião segurando o braço do membro lesionado, direcionando o mesmo para posterior, levemente superior e rotação lateral do ombro, levando à aproximação da escápula ao gradil costal com rotação lateral, superior e inclinação posterior da

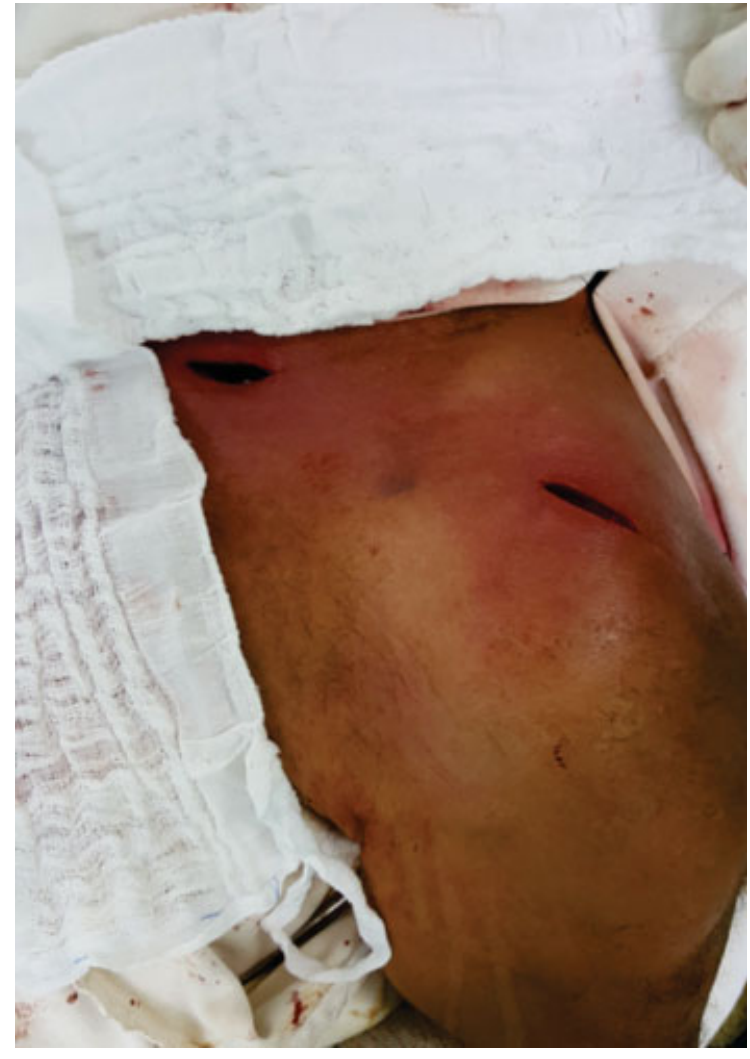

Fig. 2 Incisões medial e lateral.

escápula (posição mimetizando retração), e consequentemente reduzindo indiretamente a fratura da clavícula. Com radioscopia, verifica-se o comprimento e a forma clavicular para a escolha do tamanho do implante (placa de reconstrução de 3,5 mm não bloqueada). Após, são palpadas as extremidades medial e lateral da clavícula para localização das bordas esternal e acromial, respectivamente. É realizada, na face superior, uma incisão transversa a $1 \mathrm{~cm}$ lateral da borda esternal, de $\sim 1,5 \mathrm{~cm}$, com dissecção de planos profundos até o leito ósseo (face superior da clavícula). Na região lateral, a $1 \mathrm{~cm}$ medial da borda acromial, é realizada a segunda incisão, de igual tamanho, direção e profundidade até a face superior da clavícula. É confeccionado leito na região superior da clavícula, em sentido medial para lateral, para passagem do implante em

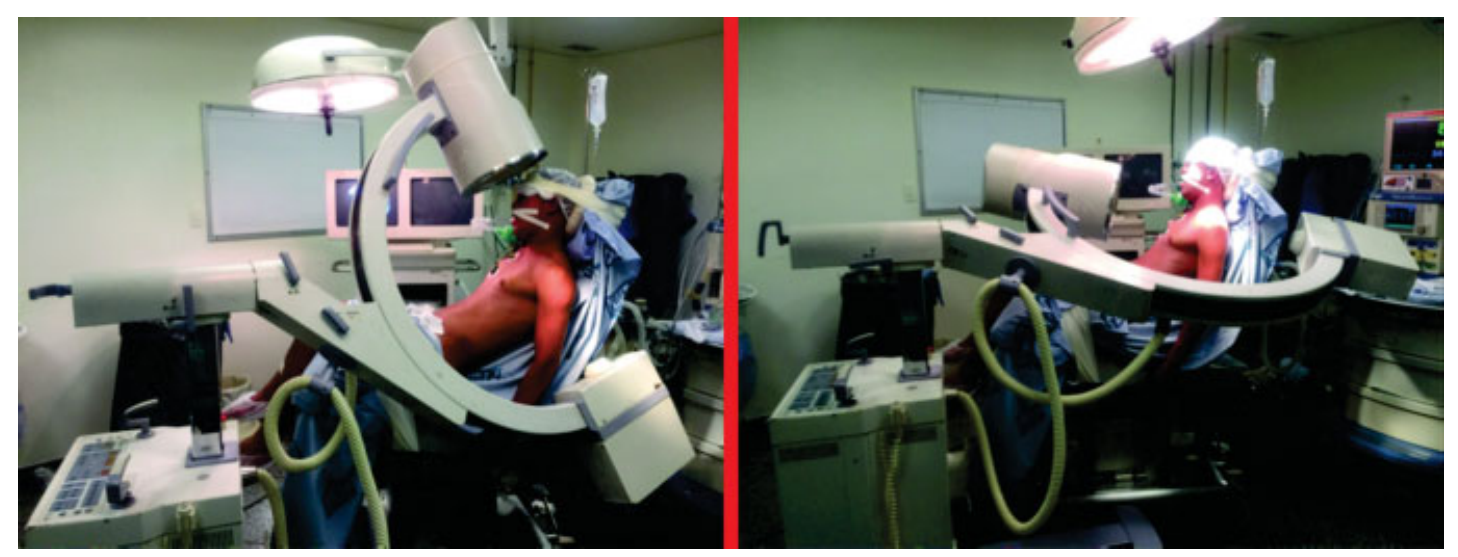

Fig. 1 Posicionamento da radioscopia em vista superior e anterior. 


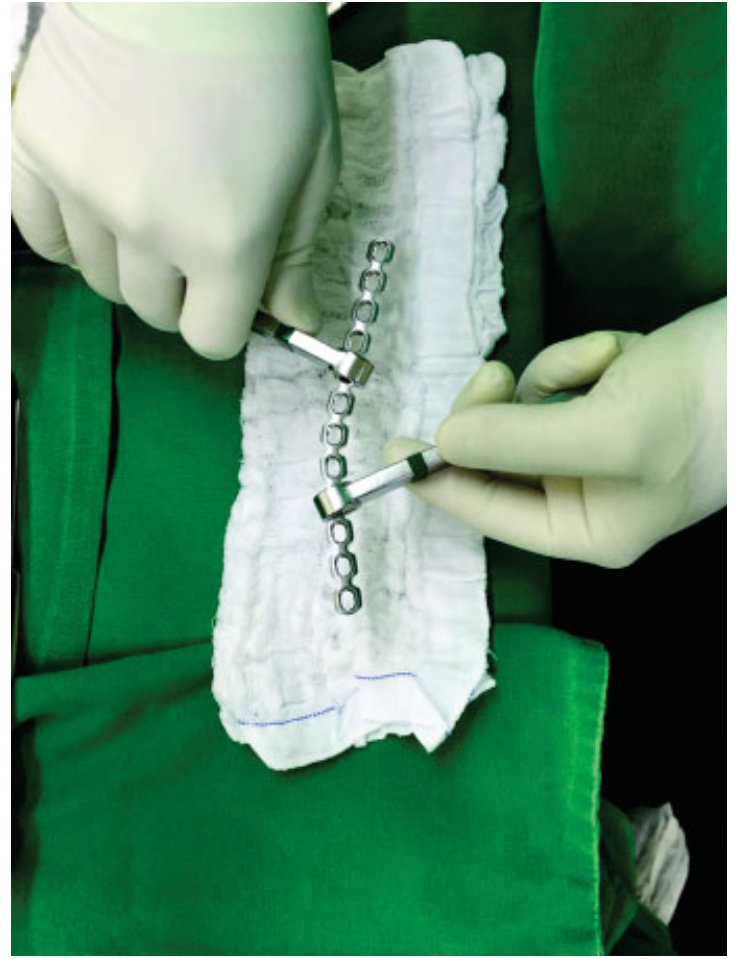

Fig. 3 Modelagem da placa.

posição superior, com instrumental para dissecção romba (-Figura 2). Modelagem da placa (-Figura 3) no intraoperatório, com convexidade anterior medial e convexidade posterior lateral, ambos ao nível do terceiro furo mais lateral e medial da placa, seguindo a forma clavicular na radioscopia. A placa é deslizada no túnel supraclavicular de medial para lateral, com a escápula mantida na posição de retração, fixação provisória com fios de Kirchner 2,5 mm (para avaliar o comprimento com auxílio da radioscopia), fixação da placa com 3 parafusos bicorticais em cada lado, de forma alternada, primeiramente pelo lado medial. É verificada a redução e o posicionamento final da placa e parafusos (-Figura 4). Feridas submetidas à irrigação com soro fisiológico $0,9 \%$, e os planos profundos foram fechados com suturas mononylon 3.0 e sutura intradérmica 2.0.

No pós-operatório, o membro foi mantido em tipoia por 6 semanas, e foram orientados movimentos plenos de cotovelo, punho e mão. Elevação, abdução $>30^{\circ}$ e rotações do ombro foram desencorajadas. Após 8 semanas, foram liberados os movimentos ativos plenos do ombro. 0 retorno às atividades com carga sobre o membro e prática de esportes foram liberados após sinais de consolidação da fratura nas radiografias de controle.

Os pacientes foram acompanhados ambulatorialmente, com retornos em 15 e 30 dias iniciais, e depois mensalmente, até que a consolidação óssea fosse verificada em controle radiográfico. A união óssea foi considerada na presença de sinais de calo ósseo em ambas as radiografias AP e cefalocaudal, e ausência de mobilidade à palpação da topografia da diáfise.

Para análise estatística, a avaliação descritiva dos dados foi expressa por tabelas de distribuição de frequências, média e desvio padrão (DP). 0 teste Exato de Fisher foi utilizado para analisar as associações entre variáveis categóricas. $\mathrm{O}$ teste $\mathrm{t}$

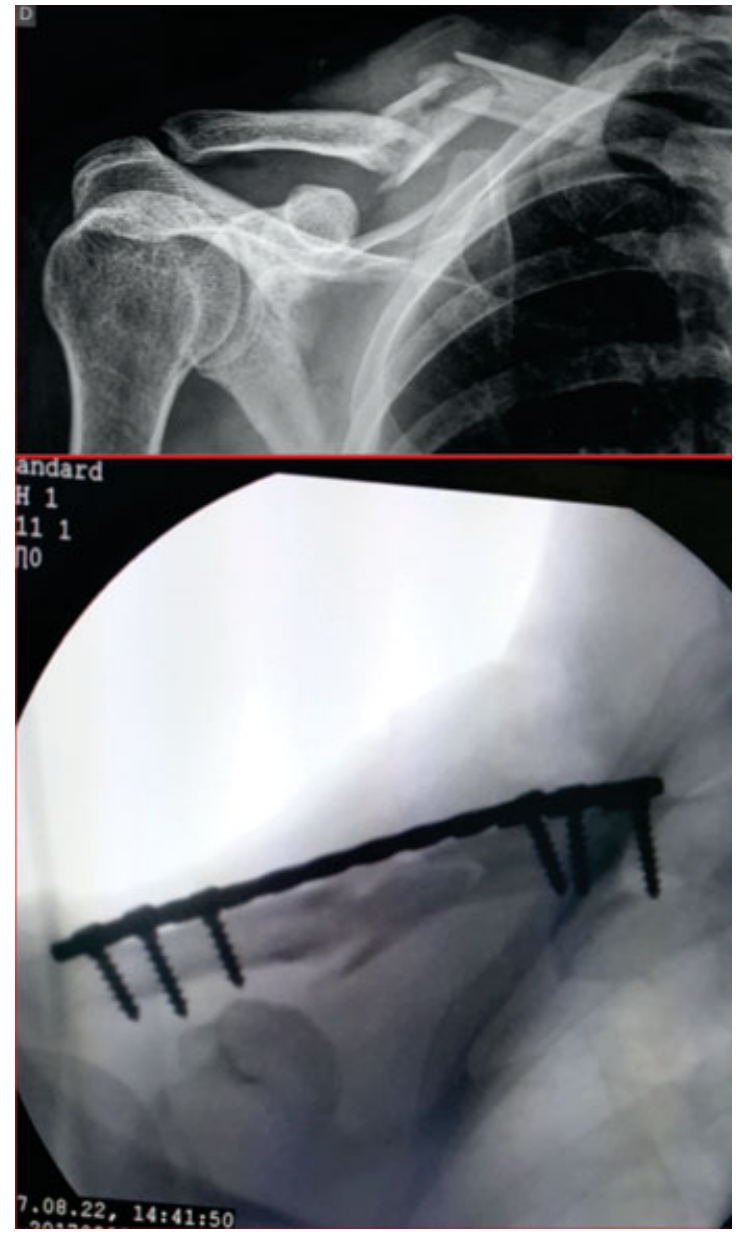

Fig. 4 Pré- e pós-fixação com técnica minimamente invasiva de osteossíntese.

pareado foi aplicado para comparar os lados operado e não operado quanto às variáveis numéricas contínuas. A normalidade dos erros foi analisada pelo box plot, gráfico quantilquantil e o teste de Shapiro-Wilk. As análises foram realizadas no programa $R^{*}$ ( $R$ Foundation, Viena, Áustria) considerando o nível de significância de $5 \%$. O presente manuscrito foi redigido de acordo com as orientações do Aprimorando a Apresentação de Resultados de Estudos Observacionais em Epidemiologia (STROBE, na sigla em inglês) para estudos observacionais (Anexo 1) e foi aprovado pelo comitê de ética da instituição sob o número CAAE 66877517.5.0000.5133.

\section{Resultados}

A amostra foi composta de 32 pacientes, sendo 31 do sexo masculino, com média de idade de 41 anos (variando de 19 a 61 anos). O seguimento médio foi de 21 meses (variando de 12 a 45 meses). As fraturas foram causadas principalmente por traumas de alta energia (acidente de moto e automóvel). O tempo médio para submeter ao procedimento foi de 9 dias. Os dados demográficos dos pacientes encontram-se na -Tabela 1.

A análise das variáveis categóricas de exame físico não mostrou diferença entre os lados operado e não operado. Não houve associação entre estas variáveis e as variáveis numéricas contínuas do exame físico ou o escore funcional. Na análise 
494 Tratamento cirúrgico da fratura multifragmentada da diáfise da clavícula Mendes Junior et al.

Tabela 1 Análise descritiva das características da amostra

\begin{tabular}{|l|l|}
\hline Variável & Frequência (\%) \\
\hline Sexo & \\
\hline Feminino & $1(3,1)$ \\
\hline Masculino & $31(96,9)$ \\
\hline Tipo de trauma & \\
\hline Acidente de moto & $14(43,8)$ \\
\hline Queda ao solo & $9(28,1)$ \\
\hline Acidente de automóvel & $4(12,5)$ \\
\hline Outros & $5(15,6)$ \\
\hline Lateralidade & \\
\hline Direito & $10(31,3)$ \\
\hline Esquerdo & $22(68,8)$ \\
\hline Dominância & \\
\hline Direito & $31(96,9)$ \\
\hline Esquerdo & $1(3,1)$ \\
\hline
\end{tabular}

entre os lados acometido pela fratura e sadio (-Tabela 2 ), observamos que o lado operado apresentou menor média de elevação passiva e maior média de comprimento da clavícula, com valores estatisticamente significantes $(p<0,05)$, comparado ao lado não operado. Não houve diferença estatisticamente significante quanto à presença ou ausência de discinesia escapular na comparação entre os lados operado e não operado.

Na avaliação funcional de DASH, seu valor médio foi de 1,75 , considerado satisfatório, e utilizando o valor de 10 pontos para análise de mínima diferença clínica significante (MCID), ${ }^{21}$ subdividimos os resultados da variável em satisfatório e insatisfatório. Os pacientes que tiveram falha precoce do método de fixação foram considerados resultados insatisfatórios para as análises de associação. Observamos que entre os pacientes com idade $>50$ anos, 33,3\% apresentaram resultado insatisfatório no DASH ( $\geq 10$ ), já entre os com idade até 50 anos, apenas $4,4 \%$ apresentaram resultado insatisfatório $(p=0,0572)$ (-Tabela 3). Dois pacientes com idade $>60$ anos $(100,0 \%)$ apresentaram resultados insatisfatórios no DASH, diferindo significativamente do grupo com idade até 60 anos $(p<0,05)$, no qual $6,7 \%$ dos pacientes tiveram resultado insatisfatório. Entre os pacientes com menor tempo de espera até a cirurgia (até 7 dias), nenhum paciente apresentou resultado insatisfatório no DASH, já entre os que esperaram $>7$ dias, 26,7\% apresentaram resultado insatisfatório $(p<0,05)$.
Tabela 3 Análise da associação das variáveis estudadas com o resultado do DASH

\begin{tabular}{|c|c|c|c|c|}
\hline \multirow[t]{3}{*}{ Variável } & \multirow[t]{3}{*}{$\mathrm{n}(\%)$} & \multicolumn{2}{|l|}{ DASH } & \multirow[t]{3}{*}{ valor-p } \\
\hline & & Insatisfatório & Satisfatório & \\
\hline & & $\begin{array}{l}\text { Frequência } \\
\text { (\%) }\end{array}$ & $\begin{array}{l}\text { Frequência } \\
\text { (\%) }\end{array}$ & \\
\hline \multicolumn{5}{|l|}{ Idade } \\
\hline $\begin{array}{l}\leq 42 \text { anos } \\
\text { (mediana) }\end{array}$ & $19(59,4)$ & $1(5,3)$ & $18(94,7)$ & 0,2788 \\
\hline$>42$ anos & $13(40,6)$ & $3(23,1)$ & $10(76,9)$ & \\
\hline$\leq 50$ anos & $23(71,9)$ & $1(4,4)$ & $22(95,6)$ & 0,0572 \\
\hline$>50$ anos & $9(28,1)$ & $3(33,3)$ & $6(66,7)$ & \\
\hline$\leq 60$ anos & $30(93,8)$ & $2(6,7)$ & $28(93,3)$ & $0,0121^{\mathrm{a}}$ \\
\hline$>60$ anos & $2(6,2)$ & $2(100,0)$ & $0(0,0)$ & \\
\hline \multicolumn{5}{|l|}{ Sexo } \\
\hline Feminino & $1(3,1)$ & $0(0,0)$ & $1(100,0)$ & 1,0000 \\
\hline Masculino & $31(96,9)$ & $4(12,9)$ & $27(87,1)$ & \\
\hline \multicolumn{5}{|l|}{$\begin{array}{l}\text { Dias para } \\
\text { cirurgia }\end{array}$} \\
\hline $\begin{array}{l}\leq 7 \text { dias } \\
\text { (mediana) }\end{array}$ & $17(53,1)$ & $0(0,0)$ & $17(100,0)$ & $0,0380^{\mathrm{a}}$ \\
\hline$>7$ dias & $15(46,9)$ & $4(26,7)$ & $11(73,3)$ & \\
\hline \multicolumn{5}{|l|}{ Queixas } \\
\hline Não & $20(62,5)$ & $1(5,0)$ & $19(95,0)$ & 0,1361 \\
\hline Sim & $12(37,5)$ & $3(25,0)$ & $9(75,0)$ & \\
\hline \multicolumn{5}{|l|}{ Consolidação } \\
\hline Não & $2(6,2)$ & $2(100,0)$ & $0(0,0)$ & $0,0121^{\mathrm{a}}$ \\
\hline Sim & $30(93,8)$ & $2(6,7)$ & $28(93,3)$ & \\
\hline
\end{tabular}

Abreviações: DASH, Disfunções do Braço, Ombro e Mão.

${ }^{\mathrm{a}}$ Teste Exato de Fisher $(p<0,05)$.

Os implantes de 12 furos foram utilizados em 28 pacientes, e a retirada foi necessária em 5 indivíduos (15,6\%). A consolidação ocorreu em 30 pacientes (93,72\%), em média de 17 semanas, e não foi observada pseudoartrose ou infecção. Como complicação, 2 pacientes tiveram falha após osteossíntese em período precoce, ambos com idade de 61 anos, soltura do implante, sem quebra da placa: um com 1 semana de pósoperatório (paciente etilista) (-Figura 5) e o outro com 8 semanas (paciente com diabetes tipo 2). Ambos foram submetidos a nova cirurgia, com redução aberta e fixação interna (RAFI) com placa e parafusos, sem uso de enxerto ósseo, e evoluíram com consolidação das fraturas.

Tabela 2 Comparação entre o lado operado e não operado quanto as variáveis analisadas

\begin{tabular}{|l|l|l|l|l|}
\hline Variável & Total Média (DP) & Lado & Nãor-p operado Média (DP) \\
\cline { 3 - 5 } & & Operado Média (DP) & $159,67(1,83)$ & $0,0497^{\mathrm{a}}$ \\
\hline Elevação (graus) & $157,67(7,89)$ & $155,67(10,73)$ & $84,50(11,17)$ & 0,4029 \\
\hline RL (graus) & $83,83(11,77)$ & $83,17(12,49)$ & $11,63(1,99)$ & 0,3662 \\
\hline Força (Kgf) & $11,43(2,51)$ & $11,23(2,96)$ & $16,13(1,22)$ & $0,0362^{\mathrm{a}}$ \\
\hline Comp. clav. (cm) & $16,23(1,23)$ & $16,33(1,26)$ & & \\
\hline
\end{tabular}

Abbreviações: Comp. clav., comprimento da clavícula; DP, desvio padrão; RL, rotação lateral.

ateste t pareado $(p<0,05)$. 


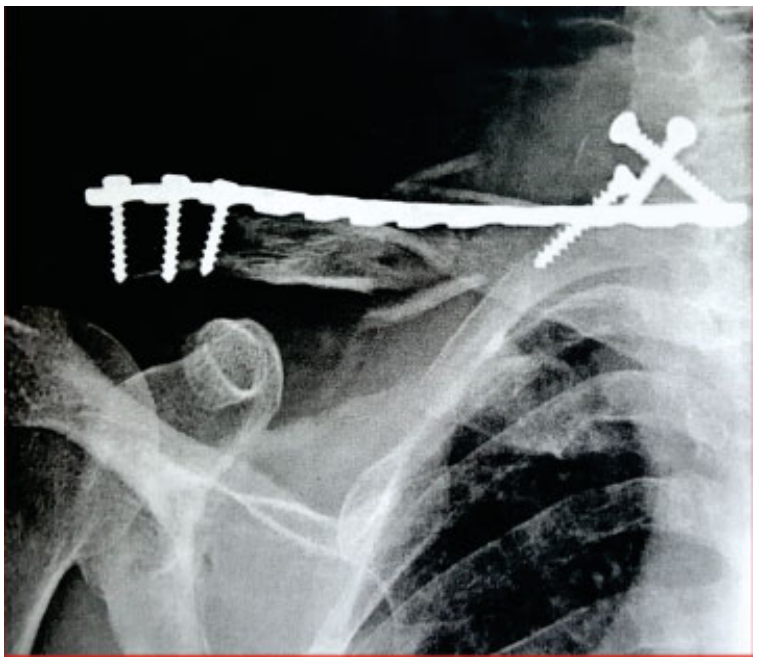

Fig. 5 Detalhamento de um dos casos com soltura precoce.

Na população em estudo, foram evidenciadas as seguintes complicações: dor aos esforços (5 pacientes - 15,6\%), desconforto sobre a placa (6 pacientes - 18,8\%), hipersensibilidade ( 2 pacientes $-6,2 \%$ ), dor em repouso ( 1 paciente $-3,1 \%$ ). Não foi relatada parestesia nas regiões das incisões cirúrgicas. Nos 2 casos de soltura precoce dos implantes, os resultados do DASH foram insatisfatórios (100,0\%), já nos casos com consolidação, foram obtidos 93,3\% de resultados satisfatórios $(p<0,05)$.

\section{Discussão}

Em nossa amostra, não obtivemos nenhum caso de pseudoartrose, tampouco de infecção. Acreditamos que a técnica descrita preserva partes moles, o foco fraturário, e contribui para a consolidação, que em nossa amostra foi de 93,72\%, semelhante ao relatado por Sohn et al. ${ }^{22} \mathrm{~A}$ amostra também apresenta bons resultados clínicos, funcionais e radiográficos, e estes estão em conformidade com a literatura. Mirzatolooei, ${ }^{4}$ comparando o tratamento cirúrgico e o conservador, encontrou no grupo de pacientes operados uma taxa de consolidação semelhante ao tratamento clínico, porém menores índices de consolidação viciosa, encurtamento e melhor DASH (média de 8,6). 0 autor descreve que utilizou método de estabilidade absoluta como escolha e fixação com placa de reconstrução em posição superior, obtendo uma pseudoartrose associada a infecção.

Os casos de falha precoce têm índice semelhante ao de Wang et al., ${ }^{23}$ que descreveram a mesma intercorrência de soltura do implante em um paciente de sua série. Nossos resultados insatisfatórios do DASH associaram-se à idade elevada e aos casos de falha precoce de fixação, o que pode nos inferir sobre a necessidade de avaliação da indicação da técnica a pacientes desta faixa etária; no entanto, devido à natureza observacional do estudo, não podemos afirmar qual fator é mais importante associado a essa complicação: a qualidade óssea ou uso de implante não bloqueado.

A técnica MIO utiliza menores incisões, e isto ébenéfico, pois evita grandes exposições que podem favorecer deiscências, infecções ou pseudoartrose. ${ }^{13}$ As incisões utilizadas nas regiões lateral e medial da clavícula não agridem as áreas de inervação dos nervos supraclaviculares, ${ }^{24}$ evitando posterior parestesia.
Outros autores corroboram os benefícios da opção minimamente invasiva. Jiang et al., ${ }^{25}$ comparando seus resultados sobre tratamento cirúrgico de fraturas multifragmentadas da clavícula entre mini-open versus RAFI, descreveram que o primeiro grupo apresentou menos disestesia, ausênciade cicatrizes hipertróficas, melhor mobilidade do ombro ipsilateral e ausência de dor. You et al. ${ }^{26}$ afirmam que seus resultados com a técnica MIO possuem menor índice de parestesia na região anterior do tórax e maior satisfação dos pacientes, comparados ao método cirúrgico tradicional.

Outra análise importante é sobre procedimentos de retirada de implante, comuns em pacientes submetidos à osteossíntese da clavícula. Nossos resultados mostraram que $15,60 \%$ da amostra necessitou de retirada do implante, índice semelhante ao de Sökücü et al., ${ }^{8}$ e menor do que o descrito por Asadollahi et al., ${ }^{27}$ de $23 \%$.

O método de redução da fratura é inédito, baseado no posicionamento da escápula em retração, descrita por Kibler et al. $^{28}$ como a posição ideal para a função do ombro. Nela, a escápula permanece, em relação ao tórax, com rotação externa e superior, inclinação posterior e translação medial. Acreditamos que a manobra contribui para o alinhamento dos fragmentos fraturados da clavícula e isso foi observado em todos os pacientes da série sistematicamente submetidos à manobra no ato operatório. Observamos ainda que, para a manutenção desta posição, não foram necessários dispositivos auxiliares para redução como fios de Kirchner, ${ }^{17}$ tração com parafuso fora da placa ${ }^{18}$ ou pequenos acessos ao foco fraturário. ${ }^{23}$ Além da manobra de retração da escápula, o implante não bloqueado posicionado superiormente é outro fator que auxilia na redução dos fragmentos, pois o uso dos parafusos corticais faz com que os fragmentos desviados inferiormente sejam levados de encontro à placa. Implantes posicionados anteroinferiormente, ou mesmo implantes com parafusos bloqueados colocados superiormente, podem não auxiliar na correção destes desvios.

Utilizamos a placa de reconstrução de $3,5 \mathrm{~mm}$, pois é um implante de fácil modelagem conforme a forma de cada clavícula. Alguns autores ${ }^{8,25,26}$ realizam a técnica MIO com implantes anatômicos ou pré-modelados, já outros ${ }^{17,18,22,23}$ compartilham nossa filosofia de modelagem individualizada da placa de reconstrução para cada caso, mas descrevem seu uso com implantes de reconstrução de 3,5 mm bloqueados. Preferimos a utilização de implantes não bloqueados pois em nosso meio são mais facilmente disponíveis, principalmente no SUS. Alzahrani et al., ${ }^{29}$ na avaliação de 102 pacientes após osteossíntese de clavícula com quatro diferentes implantes (placa de reconstrução de 2,7 mm, 3,5 mm, placa pré-moldada e placa bloqueada de 3,5 mm), afirmam que, apesar de estudos biomecânicos mostrarem diferentes propriedades de resistência, não houve diferença entre os grupos quanto à taxa de consolidação ou índice de complicações. Ressaltamos que não foram observadas quebras de implantes, fato semelhante ao descrito por Silva et al. ${ }^{30}$ ao afirmarem que no estudo sobre tratamento cirúrgico de fraturas desviadas da clavícula utilizando placa de reconstrução não bloqueada ou haste intramedular, não houve quebra da placa quando essa foi a opção de tratamento utilizada. ${ }^{30}$

O presente estudo demonstra como pontos positivos a alta reprodutibilidade da técnica, atestando sua validação interna, 
com baixas complicações, sem descrições de quebras do implante, com alta consolidação e avaliação funcional satisfatória por examinador independente. As limitações do estudo advêm de sua natureza observacional, visto que nossos controles são os resultados dos estudos semelhantes descritos por outros autores. Ainda, os autores acreditam que os indivíduos foram caracterizados com fraturas multifragmentadas de traço complexo, no entanto ressaltam que não foi feita análise dos exames radiográficos para concordância entre avaliadores sobre traço simples ou complexo, o que pode ser considerado uma fraqueza do estudo. Acreditamos, por fim, que se faz necessária a disseminação da técnica em nosso meio para validação externa e posterior avaliação, em estudos de maior nível de evidência, da mesma frente às técnicas convencionais de redução aberta.

\section{Conclusão}

A técnica MIO mostrou-se satisfatória para o tratamento da fratura multifragmentada da diáfise da clavícula, com elevada taxa de consolidação e baixo índice de complicações.

\section{Suporte Financeiro}

Não houve suporte financeiro de fontes públicas, comerciais, ou sem fins lucrativos.

\section{Conflito de Interesses}

Os autores declaram não haver conflito de interesses.

\section{Referências}

1 McKee MD. Fraturas da clavícula. In: Court-Brow CM, Heckman JD, McQueen MM, Ricci WM, Tornetta P 3rd, McKee MD, editores. Fraturas em adultos de Rockwood \& Green. 7 th ed. Rio de Janeiro: Manole; 2014:1106-1141

2 Liu W, Xiao J, Ji F, Xie Y, Hao Y. Intrinsic and extrinsic risk factors for nonunion after nonoperative treatment of midshaft clavicle fractures. Orthop Traumatol Surg Res 2015;101(02):197-200

3 Nowak J, Mallmin H, Larsson S. The aetiology and epidemiology of clavicular fractures. A prospective study during a two-year period in Uppsala, Sweden. Injury 2000;31(05):353-358

4 Mirzatolooei F. Comparison between operative and nonoperative treatment methods in the management of comminuted fractures of the clavicle. Acta Orthop Traumatol Turc 2011;45 (01):34-40

5 Rugpolmuang L, Harnroongroj T, Sudjai N, Harnroongroj T. Comminution plays no role in worsening fracture healing of conservatively treated middle third clavicular fractures. Acta Orthop Traumatol Turc 2016;50(01):32-36

6 Canadian Orthopaedic Trauma Society. Nonoperative treatment compared with plate fixation of displaced midshaft clavicular fractures. A multicenter, randomized clinical trial. J Bone Joint Surg Am 2007;89(01):1-10

7 McKee RC, Whelan DB, Schemitsch EH, McKee MD. Operative versus nonoperative care of displaced midshaft clavicular fractures: a meta-analysis of randomized clinical trials. J Bone Joint Surg Am 2012;94(08):675-684

8 Sökücü S, Menges Ö, Cetinkaya E, Parmaksızoğlu A, Kabukçuoğlu Y. Treatment of comminuted mid-diaphyseal clavicle fractures by plate fixation using a bridging technique. Acta Orthop Traumatol Turc 2014;48(04):401-405

9 Robinson CM, Goudie EB, Murray IR, et al. Open reduction and plate fixation versus nonoperative treatment for displaced mids- haft clavicular fractures: a multicenter, randomized, controlled trial. J Bone Joint Surg Am 2013;95(17):1576-1584

10 Labronici PJ, Santos Filho FCD, Reis TB, Pires RES, Junior AFM, Kojima KE. Are diaphyseal clavicular fractures still treated traditionally in a non-surgical way? Rev Bras Ortop 2017;52(04):410-416

11 Duncan SFM, Sperling JW, Steinmann S. Infection after clavicle fractures. Clin Orthop Relat Res 2005;439(439):74-78

12 Der Tavitian J, Davison JNS, Dias JJ. Clavicular fracture non-union surgical outcome and complications. Injury 2002;33(02):135-143

13 Böstman O, Manninen M, Pihlajamäki H. Complications of plate fixation in fresh displaced midclavicular fractures. J Trauma 1997; 43(05):778-783

14 Apivatthakakul T, Arpornchayanon O, Bavornratanavech S. Minimally invasive plate osteosynthesis (MIPO) of the humeral shaft fracture. Is it possible? A cadaveric study and preliminary report. Injury 2005;36(04):530-538

15 Heitemeyer U, Kemper F, Hierholzer G, Haines J. Severely comminuted femoral shaft fractures: treatment by bridging-plate osteosynthesis. Arch Orthop Trauma Surg 1987;106(05):327-330

16 Livani B, Belangero WD. Bridging plate osteosynthesis of humeral shaft fractures. Injury 2004;35(06):587-595

17 Sohn HS, Kim BY, Shin SJ. A surgical technique for minimally invasive plate osteosynthesis of clavicular midshaft fractures. J Orthop Trauma 2013;27(04):e92-e96

18 Jung GH, Park CM, Kim JD. Biologic fixation through bridge plating for comminuted shaft fracture of the clavicle: technical aspects and prospective clinical experience with a minimum of 12-month follow-up. Clin Orthop Surg 2013;5(04):327-333

19 Orfale AG, Araújo PM, Ferraz MB, Natour J. Translation into Brazilian Portuguese, cultural adaptation and evaluation of the reliability of the Disabilities of the Arm, Shoulder and Hand Questionnaire. Braz J Med Biol Res 2005;38(02):293-302

20 Smekal V, Deml C, Irenberger A, et al. Length determination in midshaft clavicle fractures: validation of measurement. J Orthop Trauma 2008;22(07):458-462

21 Roy JS, MacDermid JC, Woodhouse LJ. Measuring shoulder function: a systematic review of four questionnaires. Arthritis Rheum 2009;61(05):623-632

22 Sohn HS, Kim WJ, Shon MS. Comparison between open plating versus minimally invasive plate osteosynthesis for acute displaced clavicular shaft fractures. Injury 2015;46(08):1577-1584

23 Wang X, Wang Z, Xia S, Fu B. Minimally invasive in the treatment of clavicle middle part fractures with locking reconstruction plate. Int J Surg 2014;12(07):654-658

24 Nathe T, Tseng S, Yoo B. The anatomy of the supraclavicular nerve during surgical approach to the clavicular shaft. Clin Orthop Relat Res 2011;469(03):890-894

25 Jiang H, Qu W. Operative treatment of clavicle midshaft fractures using a locking compression plate: comparison between miniinvasive plate osteosynthesis (MIPPO) technique and conventional open reduction. Orthop Traumatol Surg Res 2012;98(06):666-671

26 You JM, Wu YS, Wang Y. Comparison of post-operative numbness and patient satisfaction using minimally invasive plate osteosynthesis or open plating for acute displaced clavicular shaft fractures. Int J Surg 2018;56:21-25

27 Asadollahi S, Hau RC, Page RS, Richardson M, Edwards ER. Complications associated with operative fixation of acute midshaft clavicle fractures. Injury 2016;47(06):1248-1252

28 Kibler WB, Sciascia A, Wilkes T. Scapular dyskinesis and its relation to shoulder injury. J Am Acad Orthop Surg 2012;20(06):364-372

29 Alzahrani MM, Cota A, Alkhelaifi K, et al. Are clinical outcomes affected by type of plate used for management of mid-shaft clavicle fractures? J Orthop Traumatol 2018;19(01):8

30 Silva FBA, Kojima KE, Silva JS, Mattar R Junior. Comparação entre o uso de placas e o de hastes flexíveis para a osteossíntese de fraturas do terço médio da clavícula: resultados preliminares. Rev Bras Ortop 2011;46(01):34-39 